\title{
Identification of Resistance Sources for Sunflower Leaf Curl Virus Disease Caused by Begomovirus
}

\author{
Deepa* and Gururaj Sunkad
}

\author{
Department of plant pathology, University of Agricultural Sciences, \\ Raichur-584104, Karnataka, India \\ *Corresponding author
}

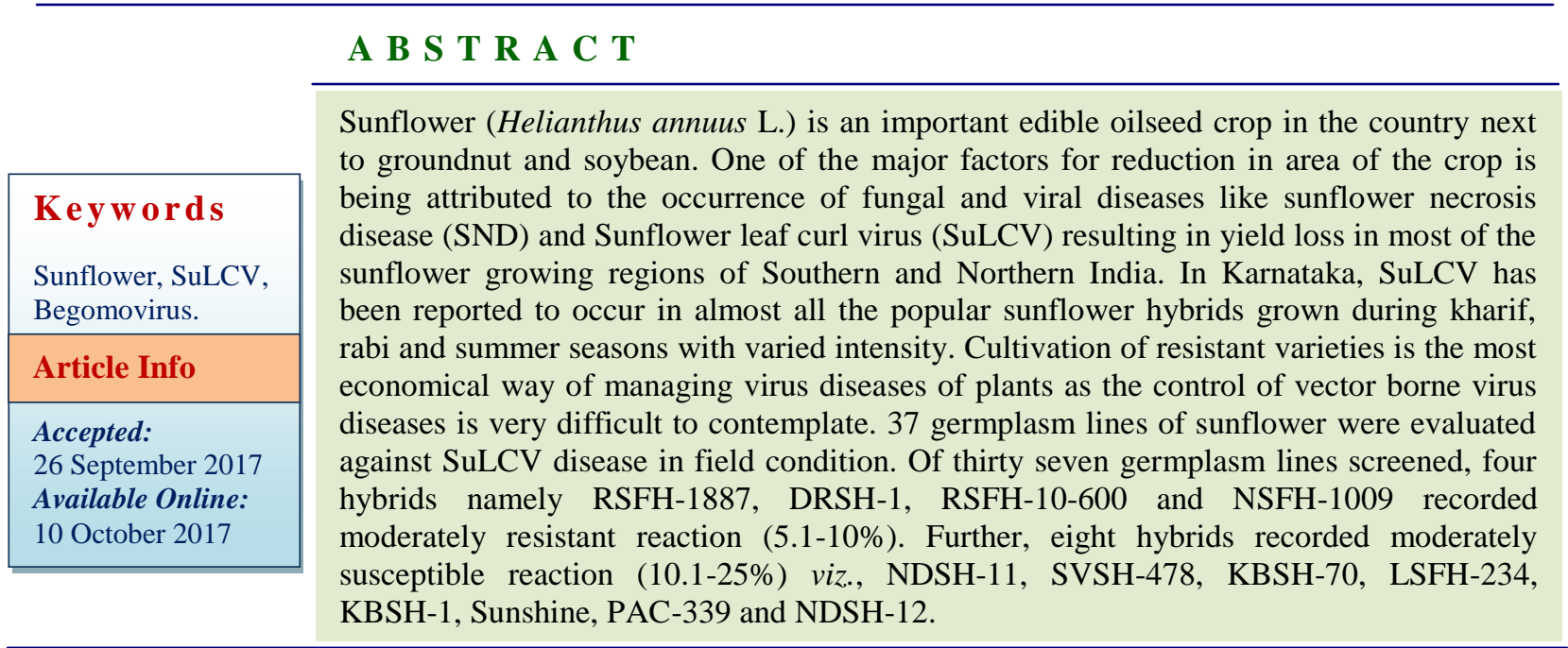

\section{Introduction}

Sunflower (Helianthus annuus L.) is one of the most important oilseed crops in India, is cultivated in an area of 0.691 million ha with an average production and productivity of 0.546 million tonnes and $791 \mathrm{~kg} \mathrm{ha}^{-1}$ respectively during the year 2013-14 (Annon, 2015). Presently Karnataka is the leading state in India contributing 64 and 54\% of total area and production respectively. It is the second important oilseed crop after groundnut in the state having an area of 0.443 million hectares with production of 0.297 million tonnes. However, productivity $\left(670 \mathrm{~kg} \mathrm{ha}^{-1}\right)$ is lesser than the national average of $791 \mathrm{~kg} \mathrm{ha}^{-1}$ (Anon., 2015). The most serious diseases of sunflower are caused by fungi and next viral diseases Necrosis and now a day's Sunflower leaf curl virus (SuLCV) becoming severe in India (Govindappa et al., 2011).

\section{Materials and Methods}

A field experiment was conducted at MARS, Raichur to identify the resistance source for sunflower leaf curl virus disease. A total 36 genotypes were received from private and public institutes at AICRP Centre, Raichur, Karnataka. These genotypes were grouped under different trials viz., initial hybrid trial (IHT), initial advanced hybrid trial (IAHT) and initial advanced hybrid trial under oleic acid (IAHT-O) and sent by Directorate of 
Oilseeds Research, Hyderabad. Each genotype was sown with a spacing of $60 \times 30$ $\mathrm{cm}$ in seven rows of $3 \mathrm{~m}$ length. The susceptible check (Morden) was sown as an infector row after each entry. The crop was raised as per the recommended package of practices except the plant protection measures against SuLCV. The screening against SuLCV was done under field condition as the disease incidence was very high during the season and observations were taken at 30 DAS, 60 DAS and 90 DAS of the crop. For recording observations, total number plants and number of SuLCV infected plants in each genotype were actually counted. Later, per cent disease was worked out by using the following formula.

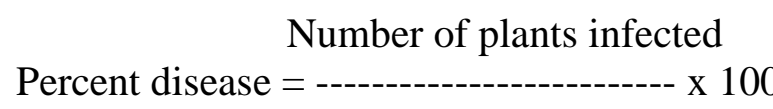

Total number of plants observed

Later, the genotypes were categorized into different categories by using $0-5$ scale on the basis of their reaction against SuLCV (Gururaj Sunkad, 2002)

\section{Results and Discussion}

Thirty six hybrids were screened for their resistance against SuLCV with morden as susceptible check. The incidence of the leaf curl virus varied from 6.94 to $75.71 \%$ in different hybrids and the susceptible check, Morden recorded an incidence of 48 per cent.

The highest incidence was observed in KSFH-280 (75.71\%), while lowest incidence was in NSFH-1009 (6.94\%) at 90 DAS.

Based on the performance of hybrids over the season, they were categorized into six reaction groups namely highly resistant ( 0 to $1 \%$ infection), resistant (1.1 to 5\% infection), moderately resistant (5.1 to $10 \%$ ), moderately susceptible (10.1 to $25 \%$ ), susceptible (25.1 to $50 \%)$ and highly susceptible $(50.1 \%$ and above).

Fig.1 Comparative differences in plants of diseased and resistance plants in screening plots

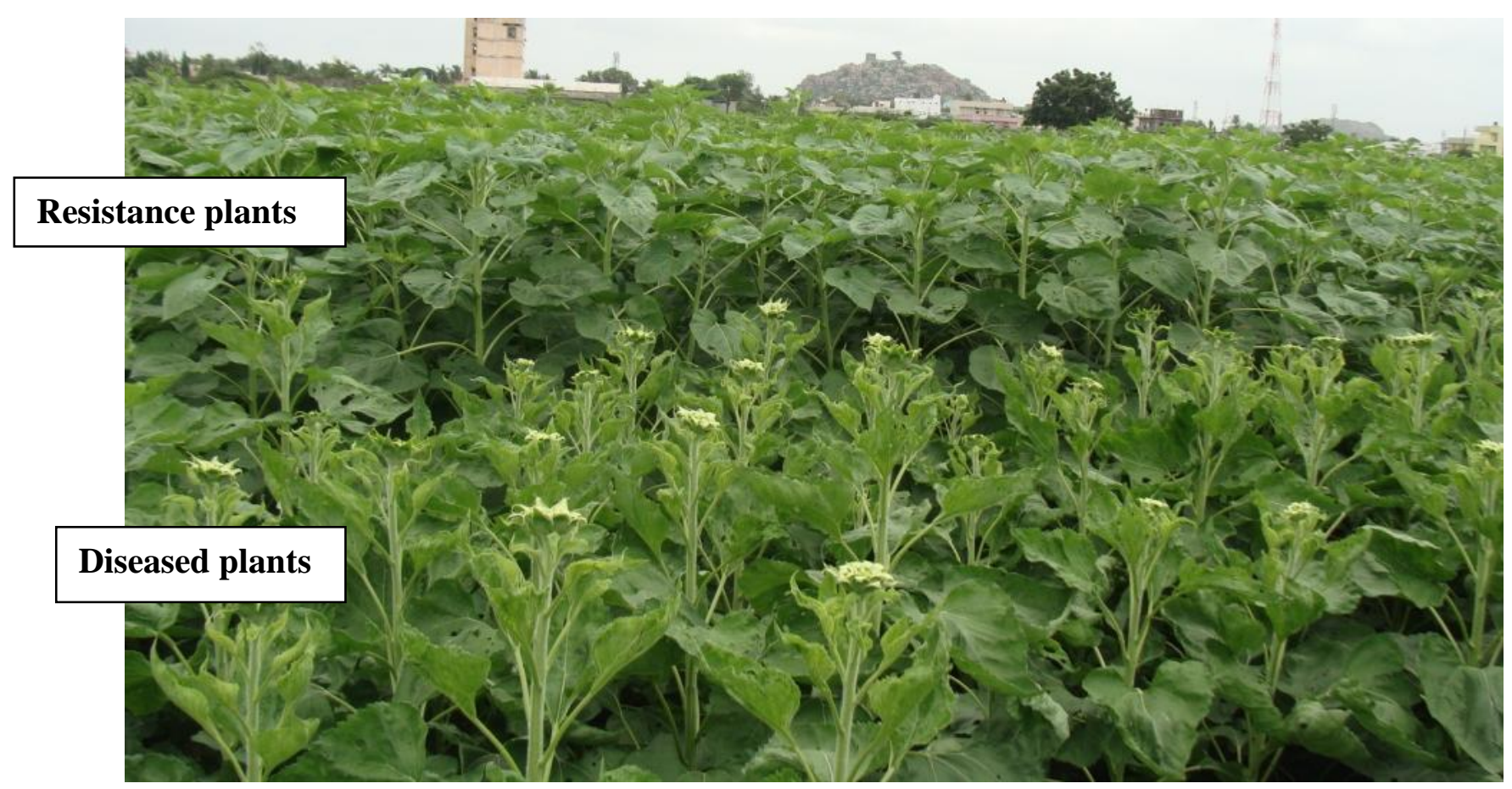


Table.1 Reaction of sunflower hybrids against sunflower leaf curl virus disease

\begin{tabular}{|c|c|c|c|c|}
\hline \multirow{2}{*}{$\begin{array}{c}\text { Sl. } \\
\text { No. }\end{array}$} & \multirow{2}{*}{ Name of the Hybrids } & \multicolumn{3}{|c|}{ SuLCV (\%) } \\
\hline & & At 30 DAS & At 60 DAS & At 90 DAS \\
\hline 1. & BRSSC-3 & 16.67 & 51.39 & 66.67 \\
\hline 2. & BSFH-111 & 32.95 & 45.00 & 58.00 \\
\hline 3. & BSFH-700 & 14.29 & 37.14 & 52.86 \\
\hline 4. & CSFH-8714 & 0.00 & 43.08 & 64.62 \\
\hline 5. & CSFH-8031 & 3.13 & 10.94 & 26.56 \\
\hline 6. & DRSH-1 & 4.55 & 6.06 & 9.09 \\
\hline 7. & DOLLER & 20.34 & 38.67 & 44.00 \\
\hline 8. & KBSH-1 & 4.35 & 14.49 & 24.64 \\
\hline 9. & KBSH-44 & 12.86 & 32.86 & 45.2 \\
\hline 10. & KBSH-68 & 24.00 & 52.67 & 68.00 \\
\hline 11. & KBSH-69 & 16.00 & 46.67 & 62.67 \\
\hline 12. & KBSH-70 & 21.43 & 15.71 & 24.29 \\
\hline 13. & KSFH-280 & 45.71 & 60.00 & 75.71 \\
\hline 14. & KSFH-284 & 20.00 & 27.14 & 62.86 \\
\hline 15. & KSFH-384 & 21.43 & 52.86 & 64.29 \\
\hline 16. & KSFH-335 & 16.42 & 50.75 & 59.70 \\
\hline 17. & LSFH-176 & 28.42 & 37.75 & 45.00 \\
\hline 18. & LSFH-234 & 0.00 & 10.77 & 21.54 \\
\hline 19. & LSFH-3436 & 14.29 & 44.29 & 74.29 \\
\hline 20. & NDSH-11 & 6.67 & 14.67 & 18.67 \\
\hline 21. & NDSH-12 & 5.71 & 15.71 & 24.29 \\
\hline 22. & NSFH-1001 & 32.90 & 43.87 & 56.00 \\
\hline 23. & NSFH-1007 & 10.00 & 41.43 & 50.80 \\
\hline 24. & NSFH-1009 & 0.00 & 0.00 & 6.94 \\
\hline 25. & NSSH-1084 & 20.55 & 39.00 & 45.25 \\
\hline 26. & NSSH-1201 & 10.00 & 22.86 & 42.86 \\
\hline 27. & PAC-339 & 0.00 & 4.62 & 13.85 \\
\hline 28. & PAC-3793 & 28.45 & 42.56 & 52.77 \\
\hline 29. & PAC-3794 & 22.00 & 37.31 & 43.71 \\
\hline 30. & PKVSH-592 & 6.67 & 21.33 & 30.67 \\
\hline 31. & PSFH-18 & 12.33 & 34.25 & 43.84 \\
\hline 32. & RSFH-10-600 & 0.00 & 2.94 & 8.82 \\
\hline 33. & RSFH-1887 & 2.67 & 6.67 & 9.33 \\
\hline 34. & SVSH-478 & 5.71 & 14.29 & 21.43 \\
\hline 35. & Sunshine & 1.54 & 4.62 & 10.77 \\
\hline 36. & VSFH-2043 & 14.25 & 23.86 & 30.00 \\
\hline 37 & Morden (Check) & 29.55 & 36.10 & 48.0 \\
\hline
\end{tabular}


Table.2 Categorization of sunflower hybrids against sunflower leaf curl virus disease

\begin{tabular}{|c|c|c|c|}
\hline $\begin{array}{l}\text { Sl. } \\
\text { No. }\end{array}$ & $\begin{array}{c}\text { Disease scale } \\
(0-5 \text { scale })\end{array}$ & $\begin{array}{l}\text { Disease grade } \\
\text { (\% incidence) }\end{array}$ & Hybrids \\
\hline 1. & 0 & $\begin{array}{l}\text { Highly resistant } \\
\quad(0 \text { to } 1 \%)\end{array}$ & - \\
\hline 2. & 1 & $\begin{array}{l}\text { Resistant } \\
(1.1 \text { to } 5 \%)\end{array}$ & - \\
\hline 3. & 2 & $\begin{array}{l}\text { Moderately resistant } \\
\quad(5.1 \text { to } 10 \%)\end{array}$ & $\begin{array}{l}\text { RSFH-1887, DRSH-1, RSFH-10-600, NSFH- } \\
1009 \text {, }\end{array}$ \\
\hline 4. & 3 & $\begin{array}{l}\text { Moderately susceptible } \\
(10.1 \text { to } 25)\end{array}$ & $\begin{array}{l}\text { NDSH-11, SVSH-478, KBSH-70, LSFH-234, } \\
\text { KBSH-1, Sunshine, PAC-339, NDSH-12 }\end{array}$ \\
\hline 5. & 4 & $\begin{array}{l}\text { Susceptible } \\
(25.1 \text { to } 50 \%)\end{array}$ & $\begin{array}{l}\text { NSSH-1201, PSFH-18, PKVSH-592, NSSH- } \\
\text { 1084, LSFH-176, VSFH-2043, PAC-3794, } \\
\text { CSFH-8031 }\end{array}$ \\
\hline
\end{tabular}

The genotypes were categorized into different categories by using 0-5 scale on the basis of their reaction against SuLCV (Gururaj Sunkad, 2002)

\begin{tabular}{|c|c|l|}
\hline Scale & Per cent disease (\%) & \multicolumn{1}{|c|}{ Disease reaction } \\
\hline 0 & $0-1$ & Highly resistant \\
\hline 1 & $1.1-5$ & Resistant \\
\hline 2 & $5.1-10$ & Moderately resistant \\
\hline 3 & $10.1-25$ & Moderately susceptible \\
\hline 4 & $25.1-50$ & Susceptible \\
\hline 5 & Above 50 & Highly susceptible \\
\hline
\end{tabular}

Out of 36 hybrids screened (Table 1) at MARS farm, none of the hybrids were found either immune or highly resistant/resistant. However, four hybrids namely RSFH-1887, DRSH-1, RSFH-10-600 and NSFH-1009 recorded moderately resistant reaction by recording less than 10 per cent SuLCV disease against the highest incidence of $48 \%$ in susceptible check variety morden. Further, eight hybrids recorded moderately susceptible reaction and they are NDSH-11, SVSH-478, KBSH-70, LSFH-234, KBSH-1, Sunshine, PAC-339 and NDSH-12. The hybrids NSSH1201, PSFH-18, PKVSH-592, NSSH-1084, LSFH-176, VSFH-2043, PAC-3794 and CSFH-8031 were found to be susceptible and rest of the sixteen hybrids were highly susceptible to SuLCV disease.

In screening, four hybrids namely RSFH1887, DRSH-1, RSFH-10-600 and NSFH-
1009 recorded moderately resistant reaction (5.1-10\%) (Table 2). Further, eight hybrids recorded moderately susceptible reaction (10.1-25\%) viz., NDSH-11, SVSH-478, KBSH-70, LSFH-234, KBSH-1, Sunshine, PAC-339 and NDSH-12. The hybrids NSSH1201, PSFH-18, PKVSH-592, NSSH-1084, LSFH-176, VSFH-2043, PAC-3794 and CSFH-8031 were found to be susceptible and rest of the sixteen hybrids were highly susceptible to SuLCV disease.

\section{References}

Anonymous, 2015. Annual Group Meeting on Sunflower, Director's report, ICARIndian Institute of Oilseeds Research.

Govindappa, M. R., Shankergoud, I., Shankarappa, K.S., Wickramaarachchi, W.A.R.T., Anjeneya Reddy, B. and Rangaswamy, K.T., 2011. Molecular 
detection and partial characterization Begomovirus associated with leaf curl disease of sunflower (Helianthus annuus) in southern India. Pl. Path. J., 10: $29-35$.
Gururaj Sunkad, Kenchanagoudar, P.V and Vijaykumar, S., 2002. Management of peanut bud vecrosis disease of groundnut usins leaf extracts. Karnataka J. Agri. Sci., 15(4): 649-652.

\section{How to cite this article:}

Deepa and Gururaj Sunkad. 2017. Identification of Resistance Sources for Sunflower Leaf Curl Virus Disease Caused by Begomovirus. Int.J.Curr.Microbiol.App.Sci. 6(10): 3226-3230. doi: https://doi.org/10.20546/ijcmas.2017.610.377 